\title{
Análise das recomendações internacionais sobre o consumo de açúcares publicadas entre 1961 e $1991^{*}$
}

\author{
An analysis of the recommendations on the consumption of sugars published throughout \\ the world between 1961 and 1991
}

\author{
Maria do Carmo M. Freire**, Geoffrey Cannon***, Aubrey Sheiham***
}

\begin{abstract}
FREIRE, M. do C.M. et al. Análise das recomendações internacionais sobre o consumo de açúcares publicadas entre 1961 e 1991. Rev. Saúde Pública, 28: 228-37, 1994. Realizou-se levantamento das recomendações nutricionais sobre o consumo de açúcares contidas nas publicações especializadas em todo o mundo, nos últimos 30 anos. Objetivou-se investigar as recomendações sobre o consumo de açúcares e identificar qual orientação deveria ser dada ao público e autoridades competentes sobre os níveis ideais de açúcares na dieta compatíveis com uma boa saúde. As informações foram obtidas de 116 publicações elaboradas por comitês de especialistas de 37 diferentes países e regiões do mundo, incluindo principalmente países desenvolvidos, mas também aqueles em desenvolvimento. Os resultados permitiram concluir que a grande maioria ( $84,5 \%$ das publicações analisadas) fazem recomendações sobre o consumo de açúcares extrínsecos ou livres, havendo consenso de que estes açúcares, principalmente a sacarose, devem ser reduzidos da dieta. A recomendação mais freqüente é $10 \%$ de calorias a partir dos açúcares extrínsecos como um nível máximo. Tais recomendações são geralınente dirigidas à toda a população com a finalidade de manter a saúde geral, mas sobretudo prevenir a cárie dental e a obesidade. Concluiu-se que as recomendações sobre o consumo de açúcares contidas nas publicações editadas nas últimas três décadas são coerentes com a evidência científica da relação entre os açúcares e as doenças crônicas e, portanto, deveriam ser integradas às políticas de alimentação e saúde.
\end{abstract}

Descritores: Necessidades nutricionais. Carboidratos. Sacarose. Educação nutricional.

\section{Introdução}

A ligação entre o fator dieta e alta prevalência de doenças crônicas tem sido objeto de crescente interesse entre cientistas e entidades governamentais, nas últimas três décadas. Desde o início dos anos 60 , comitês especializados em diversos países têm apresentadopublicações sobre recomendações nutricionais para o público e autoridades competentes, como forma de prevenir os problemas de saúde decorrentes de excessos e desvios alimentares, cada vez mais prevalentes nos países desenvolvidos.

* Síntese da Dissertação de Mestrado em Odontologia Comunitária, intitulada "An analysis of the recommendations on sugars consumption from national dietary guidelines", apresentada à "University College London Medical School", 1991.

** Departamento de Odontologia Social da Faculdade de Odontologia da Universidade Federal de Goiás, Goiânia, GO - Brasil.

*** Department of Epidemiology and Public Health of University College London Medical School - London, UK

Separatas/Reprints: M.C.M.Freire - Pça Universitária - setor Universitário - 74001-970 - Goiânia, GO Brasil
Nesse sentido, a Organização Mundial da Saúde ${ }^{137}$ recomendou em 1990 que os governantes dos países desenvolvidos e também daqueles em desenvolvimento devem estar atentos aos danos à saúde que resultam do processo de industrialização e das consequientes mudanças na dieta. Componentes específicos da dieta tais como gorduras saturadas, açúcares extrínsecos*, sal e também álcool, quando ingeridos em excesso, têm sido identificados como fatores de risco para um grande número de doenças crônicas.

Relevantes pesquisas epidemiológicas e clínicas, entre outras, têm demonstrado que o consumo de açúcares extrínsecos é uma das causas de série de doenças, especialmente a cárie dental. Segundo Sheiham ${ }^{112}$,"a cárie dental é uma doença infecciosa açúcar-dependente", uma conclusão amparada por grande parcela da evidência científica contemporânea. Uma redução do consumo de açúcares extrínsecos é considerada desejável do ponto de vista da sua cariogenicidade, bem como dos seus efeitos nocivos à saúde geral.

Análises de publicações especializadas em

\footnotetext{
* Açúcares extrínsecos são aqueles que estão fora da estrutura celular dos alimentos, incluem os açúcares livres (açúcares de mesa refinados ou naturais) adicionados aos alimentos pelo próprio consumidor ou durante o processamento de produtos como refrigerantes, confeitos, doces e outros.
} 
recomendações nutricionais têm sido realizadas desde o final dos anos 70 . O objeto de análise geralmente inclui recomendações sobre todos os nutrientes, tais como gorduras totais, gorduras saturadas, gorduras poliinsaturadas, colesterol, açúcar, carboidratos complexos, fibras, sal e álcool.

Com relação aos açúcares, a maioria das publicações recomenda uma redução do seu consumo. Contudo, nenhuma análise detalhada sobre estas recomendações tem sido apresentada até o momento.

Uma das primeiras revisões foi realizada em 1977 pelo Senado dos Estados Unidos ${ }^{109}$, que incluiu 18 publicações de vários países sobre a relação entre as gorduras da dieta e as doenças coronarianas, dos quais $44,4 \%$ (8 publicações) aconselharam redução também dos açúcares extrínsecos.

Em 1980. Turner ${ }^{128}$ revisou 20 publicações sobre alimentação e doenças coronarianas editadas num período de dez anos entre 1968 e 1978. Dos 20 trabalhos analisados. mais da metade (11) fizeram recomendações dirigidas à população em geral para reduzir o consumo de açúcares extrínsecos.

Outra revisão das recomendações nutricionais foi produzida por Mc Nutt ${ }^{71}$, em 1980, com o objetivo de identificar áreas de consenso eáreas de discordância científica que tivessem gerado confusão pública naquela época. McNutt analisou 15 trabalhos publicados entre 1957 e 1980, sendo 11 dos Estados Unidos e 4 de outros países. Em relação aos açúcares, o citado autor concluiu que apesar dos dados não sustentarem relação causal entre o consumo de açúcar e o desenvolvimento do diabetes, a maioria dos trabalhos americanos e todos aqueles dos outros países aconselham que este consumo deve ser reduzido principalmente comomeio para a prevenção da cárie dental.

Truswell ${ }^{125}$ revisou 43 publicações de 12 países e regiões do mundo publicados entre 1968 e 1982, os quais tinham como objetivo prevenir doenças coronarianas ou outras doenças crônicas. Esse autor observou que no período de 1970 a 1982 ocorreram mudanças de interesse e ênfase na questão da dieta, e foram introduzidas recomendações para se evitar o excesso de açúcares extrínsecos e álcool tendo em vista a prevenção da hipertrigliceridemia.

O'Connor e Campbell ${ }^{94}$ revisaram 49 publicações lançadas entre 1957 e 1984 principalmente nos Estados Unidos, mas também em outros países, e por organizações internacionais como a OMS e a FAO. A maioria dos trabalhos sugeria uma redução de calorias derivadas do açúcar processado e aumento do consumo de carboidratos complexos.

Trabalhos publicados entre 1976 e 1985 foram revisados por Truswell ${ }^{126}$, que encontrou resultados semelhantes àqueles obtidos em seu estudo anterior ${ }^{125}$. A limitação de açúcares extrínsecos foi aconselhada na grande maioria das publicações - 14 das 17 examinadas.

Na publicação "Dieta, Nutrição e Prevenção de
Doenças Crônicas", apresentada em 1990 pela Organização Mundial de Saúde ${ }^{137}$. foram revisados 48 importantes trabalhos especializados, publicados em países desenvolvidos e em desenvolvimento, no período de 1977 a 1990 . Das 25 recomendações oficiais para manter a saúde geral. a grande maioria (24 publicações) sugeriu redução do consumo de açúcares livres da dieta; dos 17 trabalhos destinados a baixar o risco de doenças cardiovasculares, 52,9\% (9 trabalhos) incluíam tais recomendações. Nenhum dado sobre as recomendaçōes a respeito dos açúcares foi apresentado pela OMS na revisão das 6 publicações que visavam diminuir o risco de câncer.

A mais recente e ampla revisão de trabalhos especializados foi realizada por Cannon ${ }^{34}$. Cem publicações oficiais apresentadas no período de 1961 a 1991 foram investigadas numa perspectiva histórica das alterações que têm ocorrido na dieta dos países ocidentais. Cannon concluiu que $82 \%$ das publicações analisadas recomendam consumir menos açúcar refinado, porém uma percentagem relativamente pequena destes. $28 \%$, estabeleceu metas quantitativas. Isto reflete o fato de que na maioria das publicaçðes o açúcar é menos perfilado do que as gorduras totais e gorduras saturadas. Dos trabalhos que estabeleceram metas, $74 \%$ (17 trabalhos) recomendam consumir no máximo $10 \%$ do total de calorias em açúcar, sendo esta a recomendação mais comum.

No presente esıudo são analisadas as recomendações a respeito do consumo de açúcares extrínsecos apresentadas em publicações especializadas internacionais nos últimos 30 anos, com o objetivo de verificar a relação estabelecida entre os açúcares e a prevenção das doenças crônicas, bem como identificar as possíveis recomendações sobre os níveis seguros de consumo que possam integrar a política nacional de alimentação e nutrição.

Publicaşões sobre recomendaçōes nutricionais incluidas no presente estudo

Através de revisão da literatura sobre análises realizadas por outros autores, foi possível identificar cerca de 150 trabalhos publicados no período 1961 . 1991. Deste total foram selecionados 116 para o presente estudo. excluíndo aqueles de difícil acesso, tanto pela sua localização como pela tradução.

Todos os países que editaram publicações especializadas durante este período são mencionados no presente trabalho. As publicações são provenientes de 37 países e regiões do mundo inteiro, sendo $60 \mathrm{da}$ Europa (51 do oeste, 7 do leste europeue 2 da Europa como um todo). 35 da América do Norte (32 dos Estados Unidos e 3 do Canadá), 2 da América Latina (1 do Brasil e 1 da América Latina comoum todo), 9 daOceania, 5 da Ásia, além de 5 da OMS e FAO (Tabela 1).

A grande maioria das publicações existentes nas 
Tabela 1. Número de publicações sobre recomendações nutricionais, de acordo com o país ou região de origem.

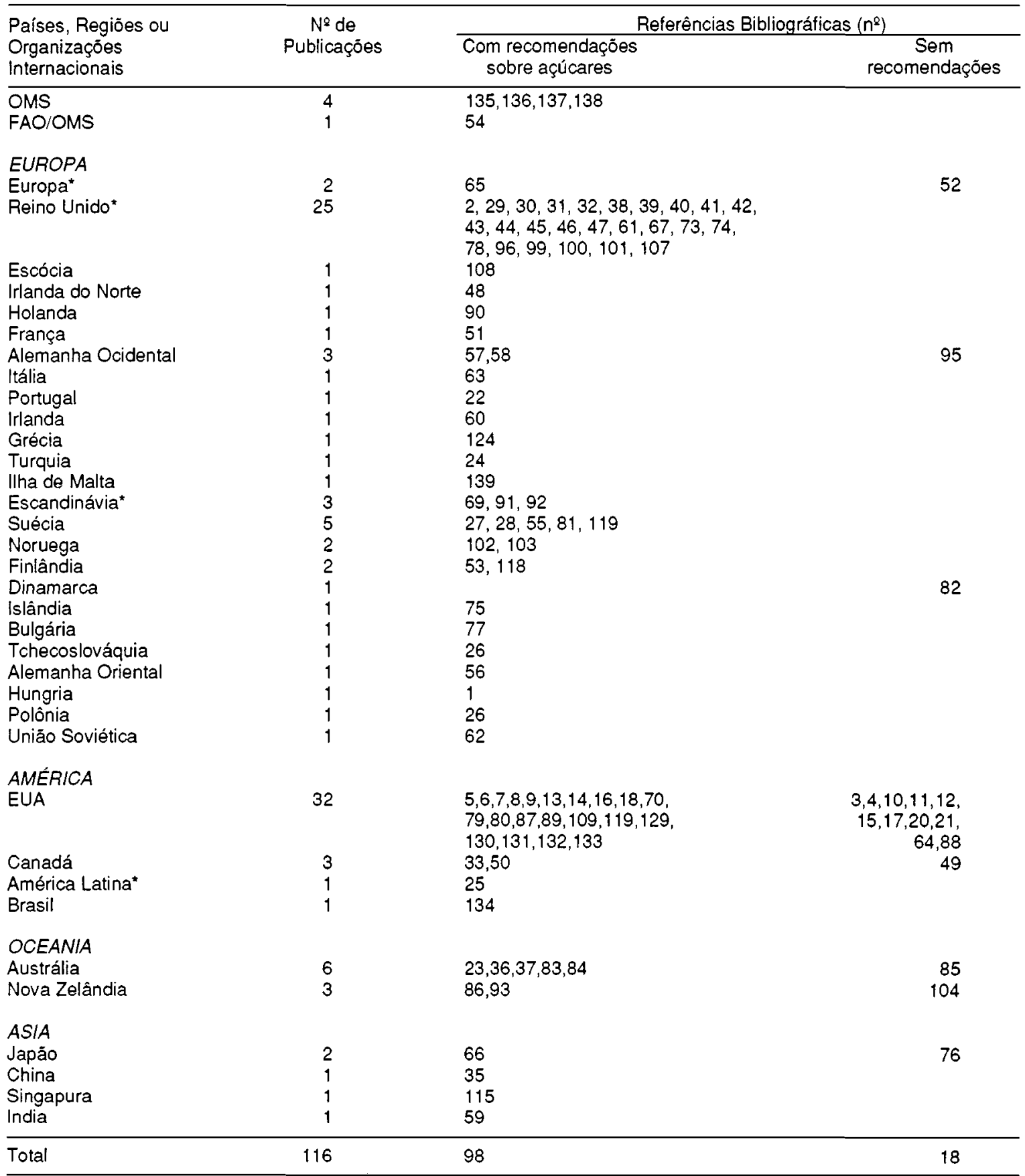

"Regiões consideradas como um todo.

últimas três décadas foi publicada em países desenvolvidos, principalmente na Europa e nos Estados Unidos. constituindo, desta forma, maioria também no presente estudo.

Os conteúdos analisados, em detalhe, referem-se aos carboidratos em geral, açúcares extrínsecos e gorduras, uma vez que as recomendações sobre os açúcares freqüentemente aparecem associados a estes outros componentes dietéticos.

As publicações foram analisadas como um todo. observando-se as seguintes informações: recomendações ao público sobre o consumo de açúcares, tipo de açúcares, quantidade e frequência de consumo, possíveis substitutos e doenças relacionadas. 
Recomendações nutricionais sobre o consumo de açúcares

A grande maioria das publicações sobre dieta elaboradas por comitês internacionais nos últimos 30 anos concordam que deve haver redução no consumo de açúcares com a finalidade de promover a saúde dos indivíduos (Tabelas 1 e 3).

Dos 116 trabalhos analisados, $84,5 \%$ (98 publicações) apresentam este tipo de recomendação (Tabela 1 ) e nenhum deles propõe aumento (Tabela 3 ).

Esta conclusão é confirmada por todos os trabalhos anteriores relatados pelo Senado dos Estados Unidos $^{109}$, Turner ${ }^{128}$, Mc Nutt ${ }^{71}$, Truswell ${ }^{125,126}$,

Tabela 2. Número de publicaçōes com recomendações sobre - consumo de açúcares extrínsecos livres ou em alimentos processados

\begin{tabular}{lc}
\hline Açúcares extrinsecos & No de publicaçōes \\
\hline Açúcares livres + & 60 \\
alimentos processados & 34 \\
Açúcares livres somente & 4 \\
Alimentos processados somente & 98 \\
\hline Total & 64 \\
Total alimentos processados & 93 \\
Total açúcares livres & \\
\hline
\end{tabular}

Tabela 3. Principais recomendaçōes sobre - consumo de açúcares extrínsecos*

\begin{tabular}{lc}
\hline Recomendaçōes encontradas & $N^{2}$ de publicaçōes \\
\hline Reduzir & 44 \\
Limitar & 6 \\
Restringir & 5 \\
Comer menos & 3 \\
Evitar comer muito & 4 \\
Evitar consumo excessivo & 2 \\
Comer raramente & 1 \\
Consumir raramente & \\
e com moderação & 1 \\
Usar somente com moderação & 2 \\
Não comer isoladamente & 1 \\
Usar apenas como condimento & 1 \\
Usar em pequenas quantidades & 3 \\
Cortar & 1 \\
Evitar & 2 \\
Limitar ou cortar & 1 \\
Excluir & 1 \\
Năo adicionar aos alimentos & \\
infantis sólidos & 1 \\
Reduzir açúcares e aumentar & \\
carboidratos complexos & 11 \\
Manter uma tendência de redução & 1 \\
Manter, Evitar que aumente & 1 \\
Não aumentar & 2 \\
\hline Total & 94 \\
\hline
\end{tabular}

Excluindo-se aqueles que se referem somente aos alimentos processados.
O'Connor e Campbel1 ${ }^{94}$, Cannon ${ }^{34}$, e pela O.M.S. ${ }^{138}$. Como pode ser observado na Tabela 2, a maioria dos trabalhos inclui recomendaçôes tanto para açúcares livres como para alimentos processados açucarados. Por outro lado, nota-se que proporção significativa refere-se apenas aos açúcares livres.

A recomendação mais comum sobre os açúcares extrínsecos livres é "reduzir" o seu consumo, estando presente em 43 publicações, de acordo com a Tabela 3 , onde são apresentadas também outras recomendações similares. Observa-se que apenas 4 trabalhos sugerem mudança mais radical na dieta com a exclusão dos açúcares extrínsecos.

Recomendações sobre os alimentos industrializados açucarados

Resultado semelhante ao da Tabela 3 foi encontrado quando foram analisadas as recomendações sobre os produtos industrializados açucarados, incluindo alimentos sólidos e líquidos. Estes produtos foram referidos em 64 trabalhos (Tabela 2) e o tipo de recomendação mais frequiente é "reduzir o consumo", sendo que nenhuma publicação aconselha aumento do consumo desses produtos.

Extensa lista de alimentos açucarados a serem reduzidos da dieta é apresentada nos trabalhos analisados, incluindo confeitos, doces e sobremesas, produtos de padaria, geléias, cereais açucarados, frutas e vegetais enlatados, molhos para saladas, maionese, "catchup", iogurtes e outros. Algumas vezes a recomendação para reduzir o consumo desses produtos açucarados aparece com o objetivo de reduzir a ingestão das gorduras que geralmente entram em sua composição.

Em relação às bebidas açucaradas, os trabalhos apontam os refrigerantes como o principal ítem a ser reduzido, mas também cafés, chás e sucos de frutas que contenham açúcar.

\section{Recomendações sobre o tipo de açúcar a ser reduzido}

Das 98 publicações que fazem recomendações sobre o consumo de açúcares, a proporção de $59,2 \%$ (58 publicações) específica o tipo de açúcar implicado na etiologia das doenças. Esta especificação é mais comum nos trabalhos mais recentes e os termos variam desde os mais genéricos, como "carboidratos simples", aos maisprecisos, como"sacarose", "glicose", "frutose" e outros.

A maior causa de preocupação é a sacarose, aparecendo em 32 trabalhos e apontada, na maioria das vezes, como o único açúcar a ser reduzido. Os açúcares não refinados, bem como o mel e xaropes manufaturados a partir do amido e largamente usados pela indústria de alimentos, também foram relatados como prejudiciais à saúde em 27 publicaçð̃es. 
Recomendações sobre quantidade efreqüência do consumo

Apesar de afirmarem que deve haver uma redução do consumo de açúcares, a maior parte dos trabalhos analisados não define metas claras e objetivas, especificando a quantidade ideal de açúcares que seria compatível com uma boa saúde.

Metasquantitativas aparecemmaisfrequientemente nos trabalhos mais atuais, o que sugere que a preocupaçãoem definir a quantidade de açúcares a ser consumida é recente nas publicações sobre recomendações nutricionais. Por outro lado, a partir de estudos como os de Takeuchi ${ }^{120,121,122}$, no início da década de 60 , já havia sido demonstrada a correlação positiva entre a incidência anual de cárie e o consumo de açúcar no Japão, quando este aumentou de $0,2 \mathrm{~kg}$ para $15 \mathrm{~kg} / \mathrm{pessoa} / \mathrm{ano}$.

Entre os trabalhos que fazem recomendações sobre açúcares extrínsecos, 24 estabeleceram metas quantificáveis para o seu consumo, seja em percentagem do consumo total de energia, seja em kg/pessoa/ ano ou g/pessoa/dia (Tabela 4). Observa-se que nenhuma meta para o consumo de açúcares extrínsecos foi observada nos trabalhos anteriores a 1977. A recomendação mais frequiente é $10 \%$ do total de energia a partir dos açúcares extrínsecos como um nível máximo, presente em 12 publicações.

O limite máximo de $10 \%$ do total de calorias a partir dos açúcares, estabelecido pela própria $O \mathrm{MS}^{137}$ e pela maioria das publicações que definem metas quantitativas, tem demonstrado ser coerente com a evidência científica que relaciona o consumo de açúcares às doenças humanas. Todavia, essas metas podem ser mais compreensíveis se, além de serem expressas em percentagem de energia ou calorias, forem também expressas em $\mathrm{g} / \mathrm{dia}$ ou $\mathrm{kg} / \mathrm{ano}$, já que estas unidades aparecem usualmente nos estudos que relacionam a quantidade de açúcares com a cárie dental. Esta relação entre percentagem de energia e peso/pessoa é estabelecida em apenas dois trabalhos (Tabela 4).

No que se refere à frequência do consumo de açúcares extrínsecos, a importância deste aspecto é reconhecida em 27 publicaçðes, havendo um consenso de que esses açúcares, tanto em sua forma livre como em produtos açucarados, devem ser evitados entre as refeições com o objetivo de prevenir especialmente a cárie dental (Tabela 5).

\section{Recomendações sobre substitutos do açúcar}

Em diversos trabalhos a recomendação para diminuir o consumo de açúcares é acompanhada de sugestões de alimentos alternativos, apontando frutas frescas e vegetais em geral, bem como outros produtos não açucarados ricos em fibra, como os melhores substitutos ${ }^{1,36,38,40,43,46,61,81,115,131}$. A substituição de açúcares extrínsecos por adoçantes artificiais não foi aconselhada com ênfase nas publicações analisadas, tendo em vista os seus prováveis efeitos tóxicos a longo prazo $1,6,26,30,32,33,46,36,63,73,78,81$.

\section{Doenças crônicas relacionadas ao consumo de açúca- res}

De acordo com os resultados obtidos no presente estudo, a cárie e a obesidade são as duas condições mais frequientemente relacionadas ao consumo de açúcares extrínsecos, juntamente com outras doenças crônicas ou fatores de risco para as mesmas, tais como diabetes, doenças coronarianas, trigliceridemia, aterosclerose, hiperlipidemia, câncer e cálculo de vesícula (Tabela 6).

Em relação à cárie, a sacarose foi fortemente identificada como o principal açúcar implicado em sua etiologia, conclusão que pode ser amparada pela extensa gama de estudos que comprovam o papel dos açúcares, sobretudo a sacarose, em sua etiologia ${ }^{105}$.

Noentanto, dadosepidemiológicostêm demonstra-

Tabela 4. Niveis recomendados de consumo de açúcares extrínsecos em \% de energia ou em $\mathrm{kg} / \mathrm{pessoa} / \mathrm{ano}$ ou g/pessoa/dia.

\begin{tabular}{lcl}
\hline Niveis de açúcares & $N^{2}$ de publicações & Referências bibliográficas (N2) \\
\hline $5 \%$ de energia & 1 & 59 \\
$10 \%$ de energia & 10 & $109,91,138,53,118,65,26,92,115,137$ \\
$10 \%$ de energia ou $20 \mathrm{~g} / \mathrm{p} / \mathrm{dia}$ & 1 & 81 \\
$10 \%$ de energia ou $60 \mathrm{~g} / \mathrm{p} / \mathrm{dia}$ & 1 & 47 \\
$12 \%$ de energia & 2 & 37,109 \\
$14 \%$ de energia & 1 & 96 \\
$15 \mathrm{a} 20 \%$ de energia* & 1 & 90 \\
$70 \mathrm{~g} / \mathrm{p} / \mathrm{dia}$ & 1 & 56 \\
$55 \mathrm{~g} / \mathrm{p} / \mathrm{dia}$ & 1 & 107 \\
$30 \mathrm{~g} / \mathrm{p} / \mathrm{dia}$ & 1 & 22 \\
$25 \mathrm{~g} / \mathrm{p} /$ dia & 1 & 30 \\
$20 \mathrm{~g} / \mathrm{p} /$ dia & 1 & 55 \\
$20 \mathrm{~kg} / \mathrm{p} / \mathrm{ano}$ & 2 & 31,78 \\
Total & 24 & \\
\hline
\end{tabular}

* Incluindo açúcares intrínsecos, que fazem parte da estrutura celular dos alimentos tais como frutas e vegetais.

* Metade ou menos em alimentos processados. 
Tabela 5. Recomendações sobre freqüência do consumo de açúcares extrínsecos

\begin{tabular}{lcl}
\hline Recomendações encontradas & Ne de publicações & Referências Bibliográficas (N8) \\
\hline Limitar ou reduzir a freqüência & 5 & $33,36,46,90,133$ \\
Evitar entre as refeiçōes & 5 & $45,83,107,131,134$ \\
Reduzir entre as refeiçōes & 7 & $27,37,57,58,69,73,78$ \\
Evitar durante e entre as refeições & 2 & 25,74 \\
Limitar às principais refeiçōes & 2 & 102,118 \\
Moderar entre as refeiçōes. Comer durante & 1 & 132 \\
Contar a frequência por dia & 1 & 63 \\
Não mais de 5 vezes/dia incluindo as refeições & 1 & 32 \\
Não mais de 3 vezes/dia & 1 & 31 \\
Não exagerar mais de 1 ou 2 vezes/semana & 1 & 1 \\
Freqüência é tão importante quanto quantidade & 1 & 47 \\
\hline Total & 27 & \\
\hline
\end{tabular}

do que nos últimos 20 anos a prevalência e severidade desta doença têm diminuído na maioria dos países desenvolvidos e aumentado rapidamente nos países em desenvolvimento, tendo como um fator relevante a disponibilidade dos açúcares ${ }^{111}$.

Este declínio da cárie tem sido associado à grande disponibilidade de cremes dentais fluoretados e às mudanças no padrão e quantidade de açúcar na dieta, entre outros fatores. Contudo, os especialistas são unânimes em afirmar que os atuais niveis de consumo ainda estão muito altos nos países desenvolvidos e uma redução ainda maior é necessária ${ }^{110}$.

Métodos não dietéticos de prevenção da cárie foram sugeridos em 30 trabalhos, todos incluindo o uso de flúor, principalmente na água de abastecimento e nos cremes dentais $19,25,27,31,32,36,37,40,42,45,46,47,49,51,56$, $65,73,74,78,89,90,93,101,103,118,131,132,133,134,137$.

Situação diferente tem sido observada nos países em desenvolvimento, onde 0 aumento do consumo de açúcares refinados, e em particular a sacarose, tem sido identificado como a principal causa dietética do aumento da prevalência da cárie ${ }^{111,112}$.

No Brasil, os níveis desta doença estão entre os mais altos do mundo, de acordo com os dados oficiais do Ministério da Saúde, em 1986 ${ }^{72}$. Além disso, a quantidade de sacarose disponível na alimentação encontra-se em torno de $137 \mathrm{~g} / \mathrm{pessoa} / \mathrm{dia}^{97}(50 \mathrm{~kg} /$ ano), uma quantidade muito acima do que seria aceitável - 40g/pessoa/dia (15 kg/ano), em presença de

Tabela 6. Doenças crônicas e fatores de risco relacionados ao consumo de açúcares extrínsecos

\begin{tabular}{lc}
\hline Doenças crônicas & No de publicaçöes \\
ou fatores de risco & 45 \\
\hline Cárie dental & 44 \\
Obesidade & 12 \\
Diabetes mellitus & 6 \\
Doenças coronarianas & 4 \\
Trigliceridemia & 3 \\
Aterosclerose & 2 \\
Hiperlipidemia & 2 \\
Câncer & 1 \\
Pedras na vesícula & \\
\hline
\end{tabular}

flúor, ou $30 \mathrm{~g} / \mathrm{pessoa} / \mathrm{dia}$ ( $10 \mathrm{~kg} / \mathrm{ano}$ ), na ausência de flúor $^{113}$ - segundo os estudos de Schulerud ${ }^{106}$, Takeuchi $^{120,121,122}$, Shimamura ${ }^{114}$, Sognnaes ${ }^{116}$, Toverud ${ }^{123}$, Knowles ${ }^{68}$, e Sreebny ${ }^{117}$.

Na ausência de qualquer política de alimentação para controlar esses altos níveis de consumo e de programas de educação em saúde bucal, que incluam as demais medidas de prevenção, tudo indica que a prevalência da cárie no Brasil tende a permanecer alta.

Sheiham ${ }^{113}$ afirma que $10 \%$ de calorias advindas dos açúcares é um nível aceitável e justificado como meta internacional, já que a maioria da população mundial não dispõe de fluoretos na água de abastecimento e nos cremes dentais.

Por sua vez, a obesidade é reconhecida como um fator de risco para uma série de doenças crônicas como diabetes, hipertensão, doenças cardiovasculares, cálculos renais e na vesícula, hiperlipidemia e câncer, tal como pode ser observado nas conclusões do Painel sobre Açúcares e Doenças Humanas do COMA Comitê sobre os Aspectos Médicos da Política de Alimentação no Reino Unido ${ }^{46}$. Essa relação indireta entre os açúcares e as doenças cardíacas parece justificar a inexistência de qualquer recomendação sobre os açúcares em 18 publicações estudadas, já que estes referem-se basicamente às doenças cardíacas e cardiovasculares (Tabela 1).

Como as doenças crônicas são as causas de mortalidade mais comuns em países desenvolvidos, medidas de saúde pública têm sido adotadas nesses países com o objetivo de minimizar o impacto dessas doenças entre as populações ${ }^{127}$. Nos países em desenvolvimento, onde as doenças decorrentes da deficiência nutricional ainda prevalecem e as doenças crônicas estão relacionadas às novas alterações na dieta, o controle destas últimas representa uma preocupação nova e crescente.

Tendo em vista a alta prevalência da cárie e o alto consumode açúcar no Brasil, decorrente da sua grande disponibilidade e baixo custo no país, e considerando os crescentes índices de doenças crônicas entre a população brasileira ${ }^{98}$, recomendaçðes consistentes para a redução do consumo de açúcares deveriam ser 
formuladas no sentido de se reverter o quadro atual.

Tais recomendações deveriam integrar-se às políticas nacionais de agricultura, alimentação e saúde e também influenciar na conduta de nutricionistas e indústrias de alimentos a fim de que se alcance uma redução do consumo a níveis inferiores a $10 \%$ do total de calorias. Uma boa prática para a promoção da saúde sem dúvida seria aumentar o consumo de amido e alimentos ricos em fibras e reduzir açúcares e gorduras.

\section{Conclusóes}

A análise das recomendações nutricionais sobre açúcares publicadas nas últimas três décadas permite concluir que:

1 - Das 116 publicações analisadas, $98(84,5 \%)$ fazem recomendações sobre o consumo de açúcares extrínsecos, havendo um consenso absoluto de que estes açúcares devem ser reduzidos da dieta tanto em sua forma livre (açúcares de mesa) como em alimentos processados;

2 - Somente 24 publicações estabelecem metas quantitativas para o seu consumo. A recomendação mais frequiente é $10 \%$ do valor energético total a partir dos açúcares extrínsecos como nível máximo;

3 - As recomendaçães para redução do consumo de açúcares extrínsecos são dirigidas à população em geral dos países desenvolvidos e em desenvolvimento. Esta estratégia tem como objetivo a manutenção da saúde, mas especialmente prevenir a cárie dental e a obesidade; 4 - A sacarose foi apontada como o principal açúcar a ser evitado em 32 publicações, tendo sido fortemente implicada na etiologia da cárie;

5 - A substituição de açúcares extrínsecos por adoçantes artificiais não é recomendada com ênfase. As alternativas apresentadas como saudáveis são as frutas frescas, vegetais em geral e outros alimentos não açucarados ricos em fibras.

FREIRE, M.do C.M. et al. [An analysis of the recommendations on the consumption of sugars published throughout the world between 1961 and 1991]. Rev. Saíde Priblica, 28: 228-37, 1994. It is study made an analysis of national expert reports on dietary guidelines published all over the world in the 30-year period between 1961 and 1991 . The aim is to identify what the reports recommend about the consumption of sugars and their relation to chronic diseases, and also what advice should be given to the public and appropriate authorities regarding levels of sugars in the diet. Information was gathered from 115 reports published by experts committees in 36 different countries and regions of the world, including developed and developing countries. The results showed that the great majority $(84,5 \%)$ of the reports analysed make recommendations on the extrinsic consumption of sugar and that there is an agreement that these sugars, especially sucrose, must be reduced in the diet. The commonest level proposed is that $10 \%$ of total calory intake from extrinsic sugars should be considered the maximun level. Such advice is often addressed to the general population and it is offered with a view to the maintenance of general heal th and especially to the prevention dental caries and obesity. It was concluded that advice on the intake of sugars given by dietary guidelines in the last three decades is coherent with the existing scientific evidence on the relationship between sugars and human diseases and, therefore, they should be integrated into national food and health policies.

Keywords: Nutritional requirements. Carbohydrats. Sucrose. Nutritional education.

\section{Referências Bibliográficas}

1. ACADEMY OF SCIENCES. Dietary guidelines for hungarians. Budapest, 1988.

2. $A D H O C$ WORKING PARTY GROUP ON CORONARY PREVENTION. Prevention of coronary heart diseases in the United Kingdom. Lancet, 1: 847, 1982.

3. AMERICAN ACADEMY OF PEDIATRICS. Prudent lifestyle for children: dietary fat and cholesterol. Pediatrics, 78: 521-5, 1986.

4. AMERICAN CANCER SOCIETY. Nutrition and cancer: cause and prevention; special report. New York, 1984.

5. AMERICAN DIABETES ASSOCIATION. Principles of nutrition and dietary recommendations for individuals with diabetes mellitus. Diabetes, 28: 1029, 1979.

6. AMERICAN DIABETES ASSOCIATION. Nutritional reconmendations and principles for individuals with diabetes mellitus: 1986. Diabetes Care, 10: 126, 1987.

7. AMERICAN Dietetic Association's nutrition recommendations for women. J. Am. Diet. Assoc., 86: 1663-4, 1986.

8. AMERICAN HEALTH FOUNDATION. Position statement on diet and coronary heart disease. Prev. Med., 1: 628-9, $277,1972$.

9. AMERICAN HEART ASSOCIATION, Central Committee for Medical and Community Program. Dietary fat and its relation to heart attacks and strokes. Circulation. 23: 123 33, 1961 .

10. AMERICAN HEART ASSOCIATION. Committee on Nutrition. Diet and heart disease. Dallas, 1965.

11. AMERICAN HEART ASSOCIATION. Committee on Nutrition. Diet and heart disease. Dallas, 1968.

12. AMERICAN HEART ASSOCIATION, Diet and coronary heart disease. Dallas, 1978.

13. AMERICAN HEART ASSOCIATION. Risk factors for coronary heart disease. Circulation. 62: 453A, 1980.

14. AMERICAN HIEART ASSOCIATION. Rationale of the dietheart statement; report of Nutrition Committee. Dallas, 1982.

15. AMERICAN HEART ASSOCIATION. Diet in the healthy child. Circulation. 67: 1411A-14A, 1983.

16. AMERICAN HEART ASSOCIATION. Inter-Society Commission for Hleart Disease Resources, special report. Circulation. 70: 188A, 1984.

17. AMERICAN HEART ASSOCIATION. Dietary guidelines for healthy american adults. Circulation. 77: 721A-24A, 1988.

18. AMERICAN HOLISTIC MEDICAL ASSOCIATION. $\mathrm{Nu}$ tritional gutidelines. 1983.

19. AMERICAN INSTITUTE FOR CANCER RESEARCH. Dictary guidelines to lower cancer risk. Washington, D.C. 1990. (AICR Information Series).

20. AMERICAN MEDICAL ASSOCIATION. Council on Food and Nutrition Diet and Coronary Heart Disease. JAMA, 222(13): 1647, 1972

21. AMERICAN MEDICAL ASSOCIATION. Concepts of nutrition and health. JAMA, 242(21): 2335-38, 1979. 
22. AMORIN CRUZ, J. Food and nutrition policy in Portugal: desirability, achievement sand constraints. In: Ferro-Luzzi, A, et al. Food and nutrition policy in mediterranean Europe. Rome World Health Organization, 1991. p.1-2. (WHO - Nutrition Series 1).

23. AUSTRALIAN ASSOCIATION OF DIETICIANS. Eating to live. Med.J. Aust., 2: 467, 1979.

24. BAYSAL, A. Food and nutrition policies in Turkey. In: FerroLuzzi A. et al. Food and nutrition policy in mediterranean Europe. Rome, World Health Organization, 1991. p.45,12 . (WHO - Nutrition Series, 1).

25. BENGOA, J.M. et al. Nutritional goals for health in Latin America. Food Nutr. Bull., 11: 10-9, 1989.

26. BERGER, S. Dietary guidelines in east european countries. In: Latham, M. \& Van Veen, M. Dietary guidelines: proceeding of an international conference, Toronto, 1988. New York, Cornell University, 1989.p. 119-21. (Comell International Nutrition Monograph Series, 21).

27. BLIX, G. \& WRETLIND, A. The national diet and a programme for its revision. Stockholm, National Institute of Public Health, 1965.

28. BLIX, G. et al. Activities in Sweden to improve dietary habits. Bibl. Nutr. Diet., 19: 159-60, 1973.

29. BRITISH DIABETIC ASSOCIATION, Dietary recommendations for diabetics for the 1980's: a policy statement. London, 1983.

30. BRITISH DIABETIC ASSOCIATION. Nutrition Sub-Committee of the British Diabetic Association's Professional Advisory Committee. Sucrose and fructose in the diabetic diet. Diabet. Med., 7: 764-9, 1990.

31. BRITISH MEDICAL ASSOCIATION. Diet, nutrition and health; report of the Broad of Science and Education. London, 1986.

32. BRITISH NUTRITION FOUNDATION'S TASK FORCE. Sugars and syrups. London, British Nutrition Foundation, 1987.

33. CANADIAN DIABETES ASSOCIATION. Guidelines for the nutritional management of diabetes mellitus: a special report. Toronto, 1984.

34. CANNON, G. Food and health: the experts agree. London, Consumer's Association, 1992.

35. CHINESE NUTRITION SOCIETY. Dietary guidelines for China. Beijing, Chinese Nutrition Society, 1989.

36. COMMONWEALTH DEPARTMENT OF HEALTH. Dietary guidelines for Australians. Camberram Australian Govermment Service, 1982.

37. COMMONWEALTH DEPARTMENT OF HEALTH. To. wards better nutrition for Atstralians; report of the Nutrition Taskforce of the Better Health Commission. Camberra, Australian Government Publishing Service, 1987.

38. CORONARY PREVENTION GROUP. Children at risk: should prevention of coronary heart disease begin in childhood? London, Coronary Prevention Group, 1988.

39. DEPARTMENT OF HEALTH AND SOCIAL SECURITY. Avoiding heart attacks. London, HMSO, 1981.

40. DEPARTMENT OF HEALTH AND SOCIAL SECURITY. Eating for health. London, 1978.

41. DEPARTMENT OF HEALTH AND SOCIAL SECURITY. Committee on medical aspects of food policy. Diet and coronary heart disease. London, HMSO, 1974.

42. DEPARTMENT OF HEALTH AND SOCIAL SECURITY. Panel on child nutrition: present day practice in infant feeding - 1980. London, HMSO, 1980.

43. DEPARTMENT OF HEALTH AND SOCIAL SECURITY. Nutrition aspects of bread and flour. London, HMSO, 1980

44. DEPARTMENT OF HEALTH AND SOCIAL SECURITY. Diet and cardiovascular disease. London, HMSO, 1984.

45. DEPARTMENT OF HEALTH AND SOCIAL SECURITY.
Panel on child nutrition: present day practice in infant feeding - third report. London, HMSO, 1988.

46. DEPARTMENT OF HEALTH. Committee on Medical Aspects of Food Policy. Dietary sugars and human disease. London, HMSO, 1989. (Report on Health and Social Subjects, 37)

47. DEPARTMENT OF HEALTH. Committee on Medical Aspects of Food Policy. Report of the panel on dietary reference value: dietary reference values for food energy and nutrients for the United Kingdom. London, HMSO, 1991.

48. DEPARTMENT OF HEALTH AND SOCIAL SERVICES. Strategy for prevention of coronary heart disease in Northern Ireland. Belfast, 1986.

49. DEPARTMENT OF HEALTH AND WELFARE. Nutrition recommmendation: a call for action. Otawa, Government Publishing Centre, 1990.

50. DEPARTMENT OF NATIONAL HEALTH AND WELFARE. Report of the Committee on Diet and Cardiovascillar Disease. Otawa, 1976.

51. DUPIN, $\mathrm{H}$. et al. Apports nutritionnels conseilles pour la population francaise. $3^{\text {eme }}$ ed. Paris, Technique et Documentation Lavoisier, 1981.

52. EUROPEAN ORGANIZATION FOR COOPERATION ON CANCER PREVENTIONSTUDIES/INTERNATIONAL UNION OF NUTRITIONAL SCIENCES. Proceedings of a joint ECP/IUNS workshop on diet and human carcinogenesis. Nutr. cancer, 8:1-40, 1986.

53. FINISH HEART ASSOCIATION. Prevention of coronary heart disease in Finland. Helsinki, 1987.

54. FOOD AND AGRICULTURE ORGANIZATION. Dietary fats and oils in human nutrition; a joint FAO/WHO report. Rome, FAO, 1977.

55. FOOD COMMITTEE. A summary of the report from the Expert Group for Diet and Health; 1983. Uppsala, Food Conmittee, 1985.

56. GERMAN DEMOCRATICREPUBLIC. Case study on nutrition policy. In: European Conference on Food and Nutrition Policy; 1st Budapest, 1990. Copenhagen, World Health Organization, 1990. p. 1-9.

57. GERMAN SOCIETY OF NUTRITION. Ten guidelines for sensible nutrition. Frankfurt, 1985.

58. GERMAN SOCIETY OF NUTRITION. Vollwertig essen und trinken nach den 10 Regeln der DGE. Frankfurt, 1989.

59. GOPALAN, C. Dietary guidelines from the perspective of developing countries. In: Lathan, M. \& Van Veen, M. Dietary guidelines: proceeding of an International Conference. Toronto. 1988, New York, Comell University, 1989. p.88, 95, 98, 100. (Comell Intemational Nutrition Monograph Series, 21).

60. HEALTH ADVISORY COMMITTEE. The prevention of coronary heart disease: diet and the food supply. Dublin, An Foras Taluntais, 1977.

61. HEALTH EDUCATION COUNCIL. Guide to healthy eating. London, 1986.

62. INSTITUTE OF PREVENTION OF NON-COMMUNICABLE DISEASES, USSR. The experience of nutrition correction in the organized population in the frame of collabo. ration with the WHO. In: European Conference on Food and Nutrition Policy, 1st Budapest, 1990. Copenhagen, World Health Organization, 1990. p.

63. INSTITUTO NAZIONALE DELLA NUTRIZIONE. Linee guida per una sana alimentazione italiana. Rome, 1988.

64. INTER-SOCIETY COMMISSION FOR HEART DISEASE RESOURCES. Primary prevention of the atherosclerotic diseases. Circulation, 42: A55-95, 1970.

65. JAMES, W.P.T. et al. Healthy nutrition; preventing nutritionrelated diseases in Europe. Copenhagen, WHO, 1988. p.96-127. (WHO Regional Publications European Series, 24). 
66. JAPAN DIETETIC ASSOCIATTON. Dietary guidelines and nutrition policies in Japan. Tokyo, 1984.

67. KINMONTH, A.L. et al. Dietary recommendations for children and adolescents with diabetes. Diabet.Med.6:537-47, 1989.

68. KNOWLES, E.M The effects of enemy occupation on the dental condition of children in the Channel Islands. Monthly Bull. Min. Health, 161-72, 1946.

69. MALMROS, G. et al. Medicinska synpunkter pa folkkosthen i de nosdiska laderna, Lakartidningen, 65.2012-3, 1968.

70. MAYER, J. Heart disease: plans for action. In: Mayer, J. US nttrition policies in the 1970. San Franscisco, W.H. Freeman, 1973. p.44, 49, 50.

71. McNUTT, K. Dietary advice to the public: 1957 to 1980 . Nutr. Rev., 38:353-60, 1980.

72. MINISTÉRIO DA SAÚDE. Levantamento epidemiológico em saúde bucal: Brasil, zona urbana, 1986. Brasilia, 1988.

73. MINISTRY OF AGRICULTURE, FISHERIES AND FOOD. Manual of nutrition. 9th ed. London, HMSO, 1985.

74. MINISTRY OF AGRICULTURE, FISHERIES AND FOOD/ DEPARTMENT OF HEALTH. Eight guidelines for a healthy diet. London, Food Sense, 1990.

75. MINISTRY OF HEALTH AND SOCIAL SECURITY. $A$ parliamentary resolution on an icelandic nutrition policy. Reykjavik, 1989.

76. MINISTRY OF HEALTH AND WELFARE. Dietary guidelines for health promotion. Nutr.Japan, 20(4):29-172, 1985.

77. NATIONAL ACADEMY OF SCIENCES. Nutrition, health promotion and chronic disease prevention: international perspectives. Proceedings of Joint US-Hungarian Conferences. Budapest, Skala Marketing Agency, 1989.

78. NATIONAL ADVISORY COUNCIL FOR NUTRITION EDUCATION. The proposals for nutrition guidelines for health education in Britain. London, Health Education Council, 1984.

79. NATIONAL CANCER INSTITUTE/NATIONAL INSTITUTE OF HEALTH. Diet, nutrition, and cancer prevention: a guide to food choices. Washington, D.C., US Govemment Printing Office, 1987. (NIH Publ., 2878).

80. NATIONAL CANCER INSTITUTE. Eating for life. Washington, D.C. USDHHS/PHS, 1988.

81. NATIONAL FOOD ADMINISTRATION. Programme of action in the area of diet and health. Eppsala, 1987.

82. NATIONAL FOOD AGENCY. The nutrition and food policy in the Danish Government. Copenhagen, 1984.

83. NATIONAL HEART FOUNDATION OF AUSTRALIA. Dietary fat and coronary heart disease: a review. Med.J. Aust., 1: 1159, 1971.

84. NATIONAL HEART FOUNDATION OF AUSTRALIA. Dietary fat and heart disease: a review. Med.J. Aust., 2: 665-6, 1974.

85. NATIONAL HEART FOUNDATION OF AUSTRALIA. Diet and coronary heart disease: a review. Med. J. Alst., 2:294-307, 1979.

86. NATIONAL HEARTFOUNDATION OFNEWZEALAND. Coronary heart disease. a New Zeeland report. John McIndoe, 1971.

87. NATIONAL RESEARCH COUNCIL. Food and Nutrition Board Assembly of Life Sciences. Towards healthful diet. Washington, D.C., National Academy of Sciences, 1980.

88. NATIONAL RESEARCH COUNCIL. Committee on Diet, Nutrition and Cancer. Assembly of Life Sciences. Diet, nutrition and cancer. Washington, D.C., National Acadenyy Press, 1982.

89. NATIONAL RESEARCH COUNCIL. Food and Nutrition Board Commission on Life Sciences. Diet and health: implications for reducing chronic disease risk. Washing. ton, D.C., National Acadeny of Sciences, 1989.

90. NETHERLANDS MINISTRY OF WELFARE, HEALTH AND CULTURAL AFFAIRS. Guidelines for a healthy diet. Den Haag, Nutrition Council, 1986.

91. NORDIC COUNCIL OF MINISTERS. Nutrition recommendations for the Nordic Countries. Oslo, 1980.

92. NORDIC COUNCIL OF MINISTERS. Nordic nutrition recommendations. Oslo, 1989.

93. NUTRITION ADVISORY COMMITTEE. Nutrition goals for New Zealanders. Health, 34: 11, 1982.

94. O'CONNOR, T.P. \& CAMPBELL, T.C. Dietary guidelines. In: Clement, I.P. et al. Dietary fat and cancer. New York, Alan R. Liss, 1986. p. 731-71.

95. PAHLKE, G. Dietary fat and degenerative vascular diseases. (Editorial). Nutr. Metb., 18: 113-5, 1975.

96. PASSMORE, R. et al. Prescription for a better British diet. $B M J, 1: 527-31,1979$

97. PINTO, V.J. A odontologia brasileira às vésperas do ano 2000: diagnóstico e caminhos a seguir. São Paulo, Santos, 1993.

98. ROUQUAYROL, M.Z. Epidemiologia e saúde. $3^{\mathrm{a}} \mathrm{ed}$. Rio de Janeiro, MEDSI, 1988.

99. ROYAL COLLEGE OFPHYSICIANSOFLONDON/BRITISH CARDIAC SOCIETY. Prevention of coronary hearth disease. J.R.Coll.Phys., 10(3): 2-34, 19-20, 1976.

100. ROYALCOLLEGE OF PHYSICIANS OF LONDON. Obesity. J.R.Coll Phys., 17(1): 51-2, 1983

101. ROYALCOLLEGEOFPHYSICIANSOFLONDON.Medical aspects of dietary fibre; a report. Tunbridge Wells, Pitman, 1980.

102. ROYAL NORWEGIAN MINISTRY OF AGRICULTURE. Norwegian nutrition and food policy; report $\mathrm{n}^{\circ} \mathbf{3 2}$ to the Storting 1975-76. Oslo, 1976.

103. ROYAL NORWEGIAN MINISTRY OF HEALTH. On the follow-lp of the Norwegian nutrition policy, report $\mathrm{n}-11$ to the Storting 1981-82. Oslo, 1982.

104. ROYAL SOCIETY OF NEW ZEALAND. Coronary heart disease. Wellington, 1971.

105. RUGG-GUNN, A.J. Diet and dental caries. In: Murray, J.J. The prevention of dental disease. Oxford, Oxford University Press, 1989. p.4-144.

106. SCHULERUD, A. Dental caries and nutrition during wartime in Norway. Oslo, Fabritius and Sonners Trykkeri, 1951.

107. SCOTTISH HEALTH EDUCATION GROUP. Eat to your heart's content. Edinburgh, 1986.

108. SCOTTISH HOME AND HEALTH DEPARTMENT. Prevention of coronary heart disease in Scotland. Edinburgh, 1990.

109. SELECT COMMITTEE ON NUTRITION AND HUMAN NEEDS. Dietary goals for the United States. Washington, US Government Printing Office, 1977.

110. SHEIHAN, A. The role of health education in reducing sugar consumption. N.Z.Dent J., 77(348): 49-56, 1981.

111. SHEIHAN, A. Changing trends in dental caries. Int. $J$. Epidemiol., 13: 142-7, 1984.

112. SHEIHAN, A. Dental caries in underdeveloped countries. In: Guggenheim, B. Cariology today. Brasil, Karger, 1984. p.33.

113. SHEIHAN, A. Why free sugars consumption should be below $15 \mathrm{~kg}$ per person per year in industrialised countries: the dental evidence. Br. Dent. J., 171: 63-5, 1991.

114. SHIMAMURA, S.A. Cohort survey on caries attacks in permenent teeth duuring a period of approximately $20 \mathrm{~kg}$ of annual sugar consumption per person in Japan. J. Dent. Health, 24: 46-52, 1974.

115. SINGAPURE MINISTRY OF HEALTH. Nationall Advisory Comnittee on Food and Nuutrition. Guidelines for $a$ healthy diet. Singapure, Ministry of Health, 1989.

116. SOGNNAES, R.F. Analysis of wartime reduction of dental caries in european children. Am. J. Dis. Child., 75; 792$821,1948$.

117. SREEBNY,LM. Sugar availability, sugar consumption and dental caries. Commumity Dent. Oral Epidemiol., 10: 1-7, 1982. 
118. STATE ADVISORY BOARD ON NUTRITION. Dietary guidelines and their scientific principles. Helsinki, 1988.

119. SWEDISH NATIONAL FOOD ADMINISTRATION. Swedish nutrition recommendations. Uppsala, 1981.

120. TAKEUCHI,M. Epidemiological study on relation between dental caries incidence and sugar consumption. Bull. Tokyo Dent. Coll., 1: 58-70, 1960.

121. TAKEUCHI,M. Epidemiological study on dental caries in Japanese children before, during and after World War II. Int. Dent. J., 11: 443-57, 1951.

122. TAKEUCHI,M. On the epidemiological principles in dental caries attack. Bull. Tokyo Dent. Coll., 3: 96-111, 1962.

123. TOVERUD, $G$. The influence of war and post-war condition on the teeth of Norwegian schoolchildren. Milbank MemFund.Quart., 35: 127-96, 1957.

124. TRICHOPOULOU, A. \& EFSTATHIADIS, P. Changes of nutrition patterns and health indicators at the population level in Greece. Am.J.ClinNutr., 49: 1042-7, 1989.

125. TRUSWELL, A.S. The development of dietary guidelines. Food Tech. Australia, 35; 498-502, 1983.

126. TRUSWELL, A.S. Evolution of dietary recommendations, goals and guidelines. Am. J.Clin Nutr., 45: 1060-2, 1987.

127. TRUSWELL, A.S. Dietary goals and guidelines in affluent countries. In: Lathan, M.C. et al. Dietary guidelines: proceedings of a international conference. Toronto, Comell International Institute, 1988. p.9-27.

128. TURNER, R. Coronary heart disease: the size and nature of the problem. Postgr. Med. J., 56: 538-47, 1980.

129. US DEPARTMENT OF HEALTH, EDUCATION AND WELFARE. Healthy people: the Surgeon-General's Report on Health Promotion and Disease Prevention. Washington, US Government Printing Office, 1979.

130. US DEPARTMENT OF AGRICULTURE. Nutrition and your health: dietary guidelines for Americans. Home Garden Bull. (April): 232, 1986.
131. USDEPARTMENTOFAGRICULTURE/USDEPARTMENT OF HEALTH. Nutrition and your health. Washington, D.C., 1980.

132. US DEPARTMENT OF AGRICULTURE/US DEPARTMENT OF HEALTH. Nutrition and your health: dietary guidelines for Americans. . Washington, D.C., 1990.

133. US DEPARTMENT OF HEALTH AND HUMAN SERVICES. The Surgeon general's Report on Nutrition and Health. Washington, US Government Printing Office, 1988.

134. VANNUCCHI, H. et al. Aplicaçöes das recomendações nutricionais adaptadas à população brasileira. Ribeirão Preto, SBAN, 1990. (Cademos de Nutrição - 2).

135. WHO EXPERT COMMITTEE ON DIABETES MELLITUS, Geneva, 1979. 2 nd report. Geneva, World Health Organization (WHO - Technical Report Series, 646).

136. WHO EXPERT COMMITTEE ON PREVENTION OD CORONARY HEART DISEASE, Geneva, 1981. Report. Geneva, World Health Organization, 1982. p. 22-3 (WHO - Technical Report Series, 678).

137. WHO EXPERT COMMITTEE ON DIET, NUTRITION, AND PREVENTION OF CHRONIC DISEASES. Geneva, WHO, 1990. P.79-80, 180-85. (WHO - Technical Report Series, 797).

138. WHO EXPERT COMMITTEE ON PREVENTION IN CHILDHOOD AND YOUTH OF ADULT CARDIOVASCULAR DISEASES, Geneva, 1988. Report. Geneva, World Health Organization, 1990. p.13, 85-6 (WHO Technical Report Series, 792).

139. WORLD HEALTH ORGANIZATION. Regional Office for Europe. Formulation of a nutrition policy; report of the First Conference on Nutrition in Malta. Copenhagen, 1986.

Recebido para publicação em 1.9 .1993

Reapresentado em 2.2.1994

Aprovado para publicacão em 11.2.1994 